\title{
Effects of Electron Concentration on the Optical Absorption Edge of InN
}

\author{
J. Wu, W. Walukiewicz*, \\ Materials Sciences Division, Lawrence Berkeley National Laboratory, Berkeley, \\ California 94720 \\ S. X. Li, R. Armitage, J.C. Ho, E.R. Weber, E.E. Haller, \\ Materials Sciences Division, Lawrence Berkeley National Laboratory, and Department of \\ Materials Science and Engineering, University of California, Berkeley, California 94720 \\ Hai Lu, William J. Schaff \\ Department of Electrical and Computer Engineering, Cornell University, Ithaca, New \\ York 14853 \\ A. Barcz and R. Jakiela \\ Institute of Physics Polish Academy of Sciences, 02-668 Warsaw, Poland
}

Molecular beam epitaxy has been used to grow InN films with free electron concentrations ranging from mid $10^{17}$ to mid $10^{20} \mathrm{~cm}^{-3}$. The optical absorption edge of these films covers a wide energy range from the intrinsic bandgap of $\mathrm{InN}$ of about $0.7 \mathrm{eV}$ to about $1.7 \mathrm{eV}$ that is close to the previously accepted bandgap of InN. The electron concentration dependence of the optical absorption edge energy is fully accounted for by the Burstein-Moss shift. Results of secondary ion mass spectrometry measurements are used to clarify the role of $\mathrm{O}$ and $\mathrm{H}$ impurities in the films.

*Electronic Mail: w_walukiewicz@lbl.gov

PACS numbers: 78.66.Fd, 71.20.Nr 
InN is an important component of the widely used group III-nitride alloys. However, it is also the least studied end-point compound of these alloys. Until recently, the bandgap of wurtzite $\mathrm{InN}$ had been believed to be about $2 \mathrm{eV}$. This value was determined from the optical absorption edge of sputter-grown InN films that had high electron concentrations $\left(>10^{19} \mathrm{~cm}^{-3}\right)$ and low electron mobilities $\left(<100 \mathrm{~cm}^{2} / \mathrm{Vs}\right)[1,2]$. Recent experiments performed on high-quality InN films grown by molecular-beam epitaxy (MBE) have shown unambiguously that the fundamental bandgap of InN is about $0.7 \mathrm{eV}[3,4]$, which is almost three times smaller than the previously accepted value. The origin of this large discrepancy is still unclear. Explanations that have been proposed include oxygen contamination that could form large-bandgap indium oxynitride in the sputter-grown films and quantum-size effects in InN nanocrystals that could raise the gap energy [3].

In previous work [5], we have noted the increase in the optical absorption edge (referred to as "optical bandgap") of InN from $0.7 \mathrm{eV}$ at a free electron concentration $(n)$ of $\sim 10^{18} \mathrm{~cm}^{-3}$ to $0.95 \mathrm{eV}$ at mid $10^{19} \mathrm{~cm}^{-3}$. This observation suggests an alternative explanation to the bandgap variation based on the free carrier doping effect. In this letter we present systematic studies of InN films grown by MBE that show various optical bandgaps ranging from $0.7 \mathrm{eV}$ to $1.7 \mathrm{eV}$. The increase in the optical bandgap is closely correlated to an increase in free electron concentration. We show that the shift of the absorption edge can be fully explained by the Burstein-Moss effect. We also discuss the relation between the electron concentration and the concentrations of $\mathrm{O}$ and $\mathrm{H}$ determined by secondary ion mass spectrometry.

Two sets of InN films were grown on sapphire substrates by MBE in two different laboratories. Details of the growth for sample set I were reported in Ref. [6]. The InN layer thickness ranged from $200 \mathrm{~nm}$ to $7.5 \mu \mathrm{m}$ [6]. The second sample set (set II) was prepared by de plasma-assisted $\mathrm{MBE}$ at substrate temperatures from $450-550^{\circ} \mathrm{C}$ and growth rates of $\sim 0.3$ microns/hour, yielding InN layers of thickness $0.5-1.0 \mu \mathrm{m}$. For the set II films the nucleation procedure began with sapphire nitridation followed by deposition of a thin $(<50 \mathrm{~nm})$ low-temperature $\mathrm{InN}$ or GaN buffer layer. Although most of the samples were not intentionally doped, free electron concentrations ranging from 
$3.5 \times 10^{17} \mathrm{~cm}^{-3}$ to $5.5 \times 10^{18} \mathrm{~cm}^{-3}$ have been found in samples of set I by Hall Effect measurements. Samples in set I that were doped with Si had even higher free electron concentrations ranging from $1.0 \times 10^{19} \mathrm{~cm}^{-3}$ to $4.5 \times 10^{19} \mathrm{~cm}^{-3}$. All the samples in set II were not intentionally doped. Free electron concentrations ranging from $3.5 \times 10^{19} \mathrm{~cm}^{-3}$ to $4.5 \times 10^{20} \mathrm{~cm}^{-3}$ were obtained by varying the residual $\mathrm{H}_{2} \mathrm{O}$ and $\mathrm{O}_{2}$ levels in the growth chamber. X-ray diffraction studies have confirmed that all $\mathrm{InN}$ samples studied were wurtzite epitaxial layers with the orientation relationship $\operatorname{InN}(0001) / / \mathrm{Al}_{2} \mathrm{O}_{3}(0001)$ or InN $(11 \overline{2} 0) / / \mathrm{Al}_{2} \mathrm{O}_{3}(10 \overline{1} 0)$. Hall mobilities range from $\sim 600$ up to $2000 \mathrm{~cm}^{2} / \mathrm{Vs}$ for the samples of set I and from $\sim 50$ to $500 \mathrm{~cm}^{2} / \mathrm{Vs}$ for the set II samples.

The optical absorption measurements were performed at room temperature using a CARY-2390 NIR-VIS-UV spectrophotometer. Secondary Ion Mass Spectrometry (SIMS) experiments were performed using a Cameca IMS 6F magnetic sector instrument with cesium primary beam and detection of $\mathrm{O}^{-}, \mathrm{H}^{-}, \mathrm{InN}^{-}$negative secondary ions. SIMS standard samples were prepared by implanting oxygen and hydrogen at controlled doses into a thick, undoped InN film $\left(n=3.5 \times 10^{17} \mathrm{~cm}^{-3}\right)$.

Figure 1 shows the absorption curves of four representative InN films. The two curves with lower absorption edges are from set I samples and the other two are from set II samples. In all cases, the absorption coefficient rises to above $5 \times 10^{4} \mathrm{~cm}^{-1}$ for a photon energy of $\sim 0.5 \mathrm{eV}$ above the absorption edge. The absorption edge energy or optical bandgap, $E_{\text {abs }}$, was determined by extrapolating the linear part of the squared absorption curves down to the baseline. The optical bandgaps obtained are plotted as a function of free electron concentration in Fig. 2.

A strong Burstein-Moss shift of the absorption edge with increasing carrier concentration can be clearly seen in Fig.2. Interestingly, the two sets of data points that cover mostly different electron concentration ranges connect very well on the plot. The optical bandgap varies continuously from $\sim 0.7 \mathrm{eV}$, the intrinsic bandgap of $\mathrm{InN}$, to $\sim 1.7$ $\mathrm{eV}$ for the sample with $4.5 \times 10^{20} \mathrm{~cm}^{-3}$ free electrons. This maximum value is close to the previously accepted value of the bandgap of $\operatorname{InN}(\sim 1.9 \mathrm{eV})$ obtained from sputter-grown, degenerately-doped InN films. Some previously reported data points adopted from the literature are also shown in Fig.2 [7]. Altogether, these data obtained from different sample sets show a consistent electron concentration dependence of the optical bandgap. 
In Ref.[5] we have shown that the lowest conduction band of InN is highly nonparabolic due to the $\boldsymbol{k} \cdot \boldsymbol{p}$ interaction across the narrow direct gap between the conduction and valence bands. The non-parabolic dispersion relation for the conduction band has been obtained from Kane's two-band $\boldsymbol{k} \cdot \boldsymbol{p}$ model [5],

$$
E_{C}(k)=E_{G}+\frac{\hbar^{2} k^{2}}{2 m_{0}}+\frac{1}{2}\left(\sqrt{E_{G}^{2}+4 E_{P} \cdot \frac{\hbar^{2} k^{2}}{2 m_{0}}}-E_{G}\right),
$$

where $E_{G} \approx 0.7 \mathrm{eV}$ is the intrinsic bandgap energy, and $E_{P}=2\left|\left\langle S\left|P_{x}\right| X\right\rangle\right|^{2} / m_{0} \approx 10 \mathrm{eV}$ is an energy parameter related to the interaction momentum matrix element. Due to the Fermi exclusion principle, optical transitions can only occur for photon energies higher than the energy needed for electrons to make vertical transitions from the valence band up to the Fermi surface in the conduction band. The increase in Fermi energy with increasing electron concentration is given by the dispersion relation in Eq.(1) evaluated at the Fermi wavevector $k_{F}=\left(3 \pi^{2} n\right)^{1 / 3}$. This approach assumes a strongly degenerate electron gas and neglects the thermal broadening of the Fermi distribution. We have calculated the absorption edge for a wide range of electron concentrations using this model. In the calculation we have also taken into account the conduction band renormalization effects due to the electron-electron interaction and the electron-ionized impurity interaction [5]. The results are in good agreement with the measured optical bandgaps, as indicated by the solid curve in Fig. 2. The dashed curve is the calculated absorption edge energy assuming a parabolic conduction band, which shows a BursteinMoss shift too fast to describe the experimental data.

It has been proposed that the large discrepancy between the previously measured large bandgap of $\mathrm{InN}$ and the recently discovered narrow bandgap could be due to the formation of indium oxynitride alloys in older sputter-grown films, since sputtered films often contain high levels of $\mathrm{O}$ contamination that can be over $10 \%$ in some extreme cases [8]. Assuming a linear composition dependence of the direct bandgap of the alloy formed by $\mathrm{InN}$ and $\mathrm{In}_{2} \mathrm{O}_{3}$ (direct bandgap $\sim 3.2 \mathrm{eV}$ [9]), we find that the highest bandgap of 1.7 eV observed in our samples would require an alloy with $40 \% \operatorname{In}_{2} \mathrm{O}_{3}$, corresponding to an oxygen concentration above $10^{22} \mathrm{~cm}^{-3}$. Clearly such high levels of $\mathrm{O}$ contamination are not expected in MBE grown InN and are highly unlikely even for sputtered samples. 
To further elucidate the origin of bandgap variation, we have performed SIMS experiments on selected, not intentionally doped films. In addition to oxygen [10], hydrogen $[11,12]$ has also been suggested as a possible donor in InN. Considering the growth process, these two elements are the most likely candidates among possible donor contaminants. In the inset of Fig.3, a set of SIMS curves obtained from a film with $n=$ $5.5 \times 10^{19} \mathrm{~cm}^{-3}$ is shown. It can be seen that both the $\mathrm{O}$ and $\mathrm{H}$ concentrations are lower than the free electron concentration. The average concentration is obtained by integrating the concentration curve over the depth and dividing by the film thickness. In Fig. 3 the average $\mathrm{O}$ and $\mathrm{H}$ concentrations are compared with electron concentration for all measured samples. There is an overall trend of higher $\mathrm{O}$ and $\mathrm{H}$ concentration for the samples with larger free electron concentration. In fact, in the most heavily doped samples the $\mathrm{O}$ concentration is, within experimental error, equal to the electron concentration. However it is also evident that in some instances the concentrations of $\mathrm{O}$ and/or $\mathrm{H}$ are too low to account for the electron concentration. This indicates that intrinsic defects such as $\mathrm{N}$ vacancies [10] and/or dislocations [13] could be important additional sources of free electrons in these samples. It is important to note that in all films, the $\mathrm{O}$ concentration is far too low to explain the increase in the bandgap purely by InN-In ${ }_{2} \mathrm{O}_{3}$ alloying effect. Therefore, we can safely exclude alloying as the origin of the large optical bandgap variations in InN [14]. We also note that in a recent paper [14] it was reported that the optical bandgap of poly-crystalline $\mathrm{InN}$ increases from $\sim 1.6 \mathrm{eV}$ to $\sim 2.2 \mathrm{eV}$ for oxygen concentrations ranging from $1 \%$ to $6 \%$. Assuming that all the oxygen atoms are electrically active substitutional donors in those samples, their data points agree reasonably well with the calculated curve shown in Fig. 2.

Our study shows that the electron concentration can vary by orders of magnitude in nominally undoped MBE grown InN. This striking propensity for n-type doping can be attributed to the extremely large electron affinity of $\mathrm{InN}$. The conduction band of $\mathrm{InN}$ is located at about $5.7 \mathrm{eV}$ below the vacuum level or $0.8 \mathrm{eV}$ below the Fermi level stabilization energy $\left(\mathrm{E}_{\mathrm{FS}}\right)$ that represents the average energy of the dangling bonds in semiconductors [15]. The unusually low location of the conduction band edge strongly favors incorporation of donor impurities and formation of donor-like native defects. According to the amphoteric defect model [16], the dangling bond defects have a donor- 
like character until the Fermi level reaches $E_{F S}$. At this point the formation energies of donor- and acceptor-like native defects become equal, resulting in a stabilization of the Fermi energy. This assertion has been recently confirmed by a study of free $\mathrm{InN}$ surfaces indicating that indeed the surface Fermi level pinning position is stabilized at about 0.8 above the conduction band edge [17].

In conclusion, we have grown and characterized $\mathrm{InN}$ films with a wide range of electron concentrations. The optical absorption edge energy of these films strongly depends on the electron concentration and varies between $0.7 \mathrm{eV}$ and $1.7 \mathrm{eV}$. The variation in the absorption edge can be fully explained by the effect of Fermi level increase as a result of free electron doping. An analysis of the SIMS results shows that oxygen and hydrogen doping cannot fully account for the free electron concentration, suggesting an important role of native defects. Also the results exclude the possibility that $O$ alloying effects could explain the observed changes of the absorption edge energy.

After the completion of the manuscript, we noted a most recent paper by Bhuiyan et. al. that relates the variation of absorption edge of InN to the Burstein-Moss Effect [18].

We thank Dr. K. M. Yu and Mr. J. W. Beeman for preparing the standard sample for SIMS. This work was supported by the Laboratory Directed Research and Development Program of Lawrence Berkeley National Laboratory under the Department of Energy Contract No. DE-AC03-76SF00098. The work at Cornell University is supported by ONR Contract No. N000149910936. 
References

[1] T. L. Tansley and C. P. Foley, J. Appl. Phys., 59, 3241 (1986).

[2] W. Z. Shen, L. F. Jiang, H. F. Yang, F. Y. Meng, H. Ogawa and Q. X. Guo, App. Phys. Lett. 80, 2063 (2002).

[3] V. Yu. Davydov, A. A. Klochikhin, R. P. Seisyan, V. V. Emtsev, et. al., phys. stat. solidi (b), 229, R1 (2002).

[4] J. Wu, W. Walukiewicz, K. M. Yu, J. W Ager III, E. E. Haller, Hai Lu, William J. Schaff, Yoshiki Saito and Yasushi Nanishi, Appl. Phys. Lett., 80, 3967(2002).

[5] J. Wu, W. Walukiewicz, K. M. Yu, J. W. Ager III, E. E. Haller, Hai Lu and William J. Schaff, Phys. Rev. B. 66, R201403 (2002).

[6] Hai Lu, William J. Schaff, Jeonghyun Hwang, Hong Wu, Goutam Koley and Lester F. Eastman, Appl. Phys. Lett. 79, 1489(2001).

[7] V. A. Tyagai, et. al., Sov. Phys. Semicond. 11, 1257 (1977); T. Inushima, et. al., Inst. Phys. Conf. Ser. No. 142: Chapter 5, p.971; T. Inushima, et. al., Solid State Commun., 110, 491 (1999); D. B. Haddad, et. al., unpublished.

[8] M. Winterbert-Fouquet, K. Butcher and Motlan, phys. stat. sol. (c), 0, 2785 (2003).

[9] D. Mergel and Z. Qiao, J. Phys. D: Appl. Phys. 35, 794 (2002).

[10] C. Stampfl, C. G. Van de Walle, D. Vogel, P. Krueger and J. Pollmann, Phys. Rev. B, 61, R7846(2000).

[11] D. C. Look, H. Lu, W. J. Schaff, J. Jasinski and Z. Liliental-Weber, Appl. Phys. Lett., 80, 258 (2002).

[12] E. A. Davis, S. F. Cox, R. L. Lichti, C. G. Van de Walle, Appl. Phys. Lett., 82, 592 (2003).

[13] H. Lu, W. J. Schaff, L. F. Eastman, J. Wu, W. Walukiewicz, D. C. Look and R. J. Molnar, Mater. Res. Soc. Proc., 743, L4.10 (2002).

[14] M. Yoshimoto, et. al., Appl. Phys. Lett., 83, 3480 (2003).

[15] J. Wu, W. Walukiewicz, K. M. Yu, J. W. Ager III, E. E. Haller, Hai Lu, William J. Schaff, W. K. Metzger and Sarah Kurtz, J. Appl. Phys., 94, 6477 (2003).

[16] W. Walukiewicz, Appl. Phys. Lett., 54, 2094(1989). 
[17] I. Mahboob, T. D. Veal, C. F. McConville, H. Lu and W. J. Schaff, unpublished.

[18] A. G. Bhuiyan, K. Sugita, K. Kasashima, A. Hashimoto, A. Yamamoto and V. Yu. Davydov, Appl. Phys. Lett. 83, 4788 (2003). 


\section{Figure Captions}

Fig. 1 Absorption spectra of InN films with different free electron concentrations.

Fig. 2 Energy of optical absorption edge as a function of free electron concentration covering three decades of change. Some previous data points are also shown [7]. The solid curve shows the calculated optical absorption edge.

Fig. 3 Free electron concentration shown in comparison with oxygen and hydrogen concentrations. The inset shows the SIMS data of an InN film with $n=5.5 \times 10^{19} \mathrm{~cm}^{-3}$. 


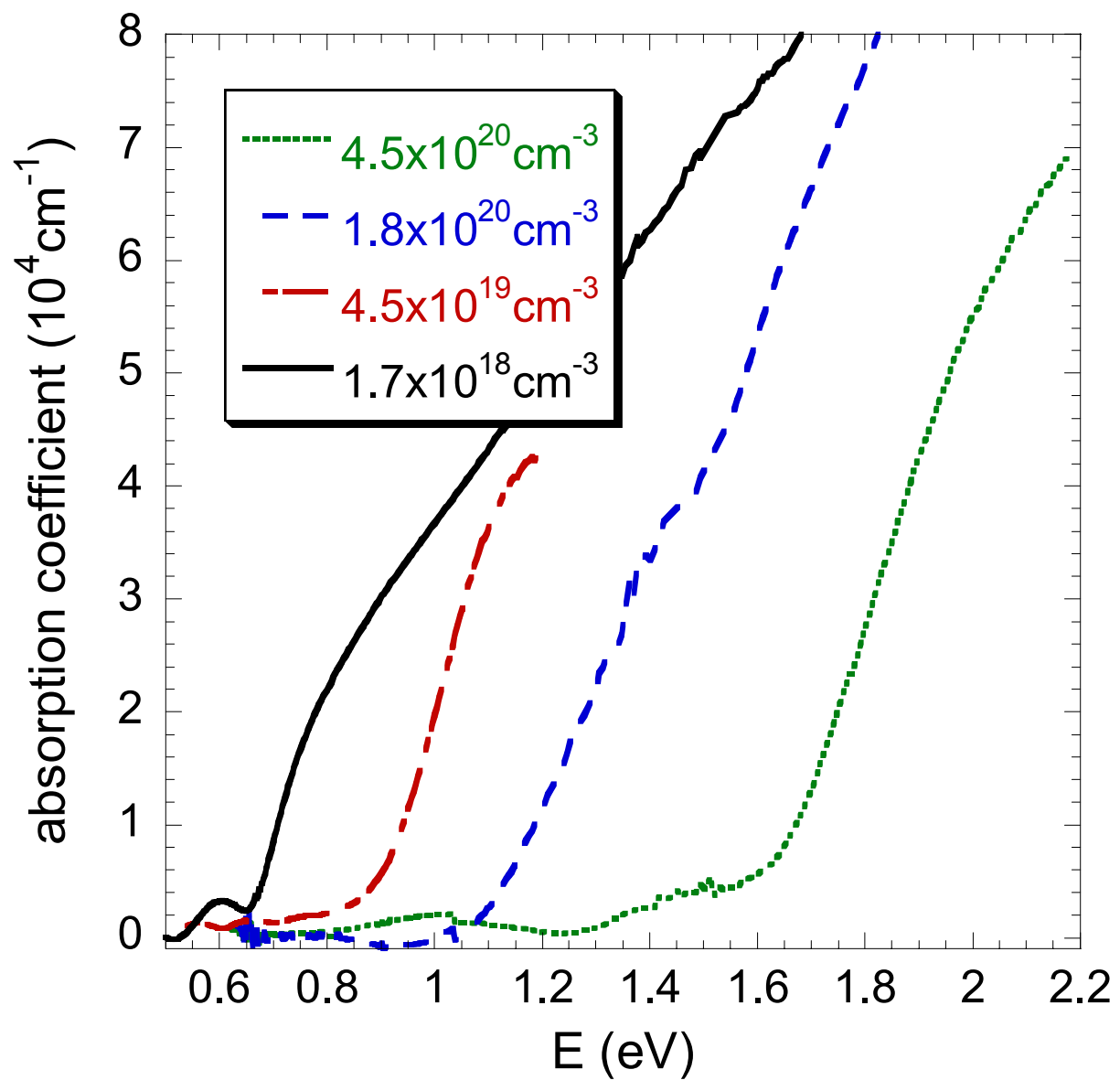

Fig. 1 of 3

$\mathrm{Wu}$ et. al. 


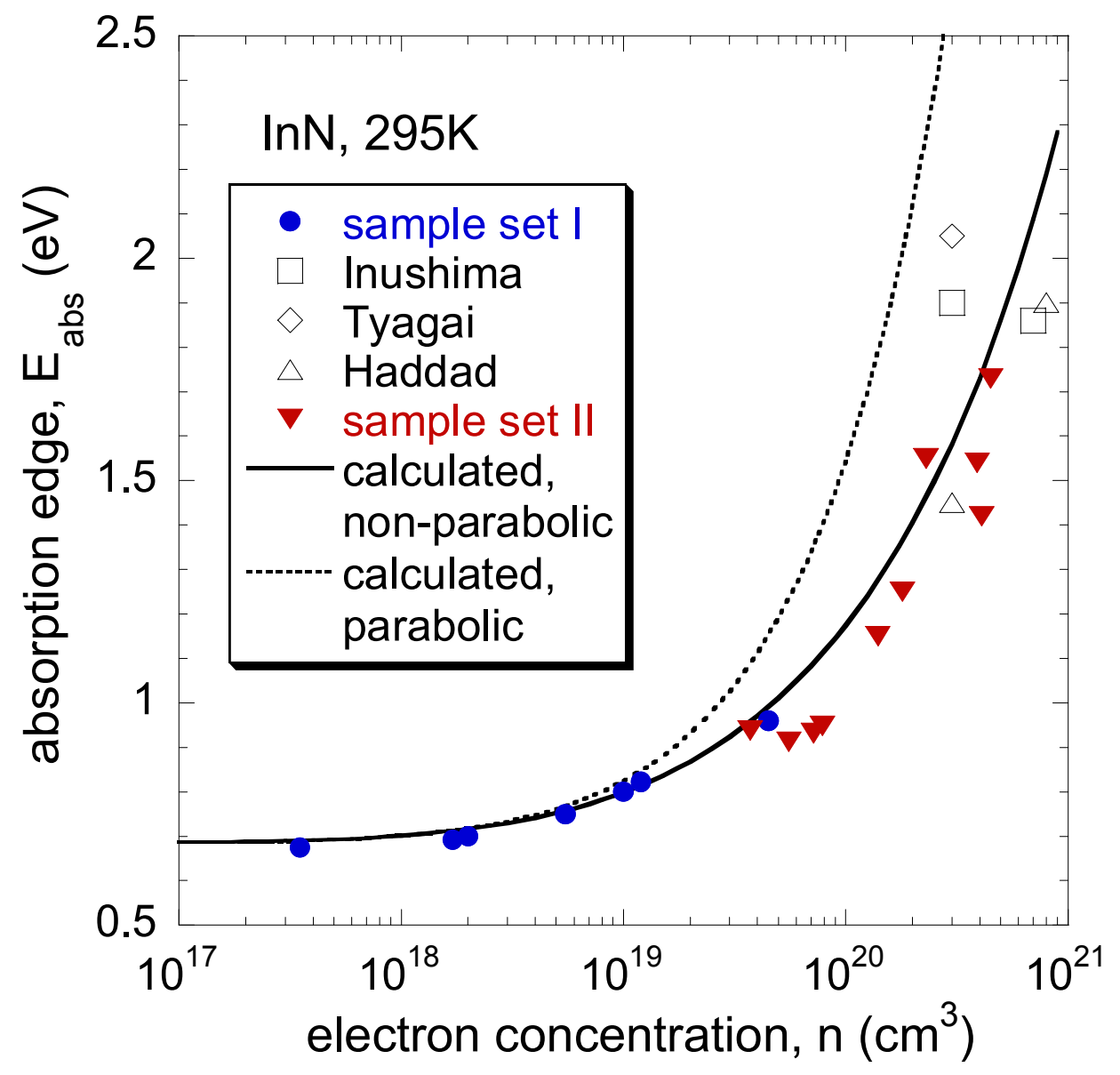

Fig. 2 of 3

Wu et. al. 


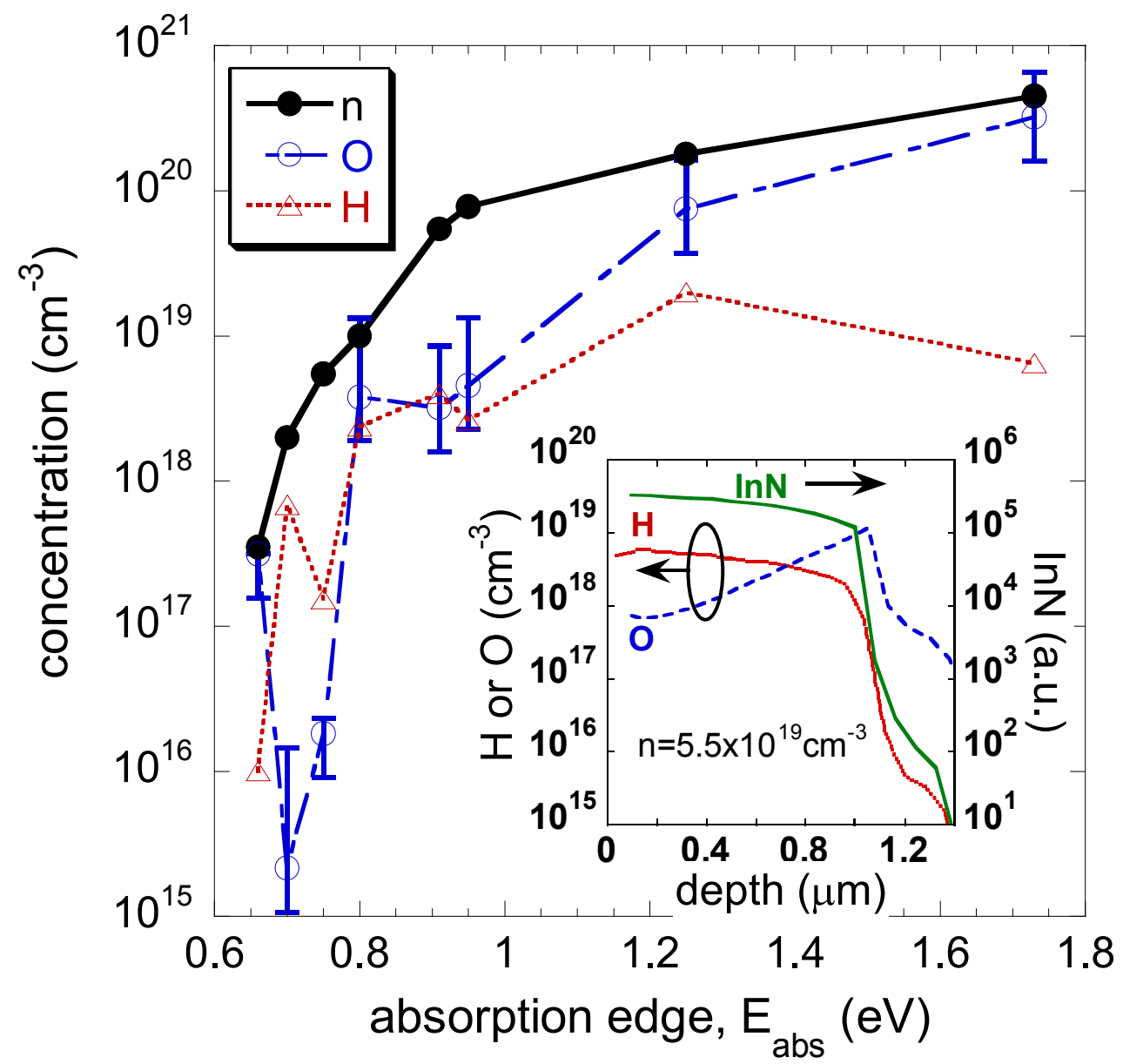

Fig. 3 of 3

Wu et. al. 